\title{
Câncer bucal na percepção dos acadêmicos dos cursos de saúde da UninCor/Três Corações -MG
}

\author{
Oral cancer in the perception of the academics \\ of UNINCOR/Três Corações -MG health courses \\ Percepción de académicos de cursos de salud \\ de la UNICOR/ Três Corações-MG sobre el cáncer oral \\ Érica de Andrade CARVALHO' \\ Marina Santana GAZOLLA ${ }^{2}$ \\ José Dias da SILVA NETO ${ }^{2}$ \\ Ana Paula de Oliveira MACIEL2 \\ Kamila Prado Pereira GRACIANO ${ }^{3}$ \\ Jessica Luana DOS SANTOS ${ }^{4}$ \\ Jorge Esquiche LEÓN ${ }^{5}$ \\ Matheus Henrique Lopes DOMINGUETE ${ }^{6}$ \\ ${ }^{I}$ Acadêmica do Curso de Odontologia, Universidade Vale do Rio Verde de Três Corações-UninCor, \\ 37410-000 Três Corações-MG, Brasil \\ ${ }^{2}$ Professor (a) do Curso de Odontologia, Departamento de Diagnóstico e Cirurgia Oral, \\ Universidade Vale do Rio Verde de Três Corações-UninCor, 37410-000 Três Corações-MG, Brasil \\ ${ }^{3}$ Mestre em Odontologia, Departamento de Diagnóstico e Cirurgia Oral, \\ Universidade Vale do Rio Verde de Três Corações-UninCor, 37410-000 Três Corações-MG, Brasil \\ ${ }^{4}$ Doutoranda, Laboratório de Histopatologia e Microscopia, Faculdade de Odontologia de Ribeirão Preto, \\ Universidade de São Paulo-USP, 14040.904 Ribeirão Preto-SP, Brasil \\ ${ }^{5}$ Professor Doutor, Faculdade de Odontologia de Ribeirão Preto, Universidade de São Paulo-USP, \\ 14040.904 Ribeirão Preto-SP, Brasil \\ ${ }^{6}$ Professor Orientador, Departamento de Diagnóstico e Cirurgia Oral, \\ Universidade Vale do Rio Verde de Três Corações-UninCor, 37410-000 Três Corações-MG, Brasil
}

\begin{abstract}
Resumo
Objetivo: Avaliar o conhecimento de estudantes de diferentes cursos da área da saúde da Universidade Vale do Rio Verde de Três Corações (Minas Gerais, Brasil) sobre o câncer de boca. Método: Foi realizado um estudo observacional, no qual 314 estudantes dos cursos de farmácia, enfermagem, nutrição e odontologia responderam a um questionário estruturado com 17 perguntas divididos entre autoavaliação e caraterísticas objetivas sobre o câncer de boca. Resultados: Uma parcela representativa da população universitária sabe que o câncer pode acometer a boca e que o tabagismo e etilismo são fatores de risco, entretanto, mostraram desconhecimento sobre a faixa etária mais afetada. Notou-se que os 4 cursos apresentaram nível de conhecimento deficiente, contrapondo-se ao questionário de auto avaliação. Houve uma tendência de alunos do primeiro e segundo anos da graduação obterem pontuação maior. Conclusão: Os dados obtidos sugerem a necessidade de estratégias direcionadas ao processo ensino/aprendizagem, visando o melhor aproveitamento e fixação do conhecimento acerca do câncer de boca, bem como o enfoque no desenvolvimento de políticas de prevenção desta doença no projeto pedagógico destes cursos.
\end{abstract}

Descritores: Neoplasias Bucais; Saúde Bucal; Centros Educacionais de Áreas de Saúde; Avaliação Educacional.

\begin{abstract}
Introduction: To evaluate the knowledge of students from different health courses at the Vale do Rio Verde University of Três Corações (Minas Gerais, Brazil) on oral cancer. Methods: An observational study was conducted in which 314 students from the pharmacy, nursing, nutrition and dentistry courses answered a structured questionnaire composed of 17 questions. The questions were divided in two parts: self-evaluation and objective characteristics about oral cancer. Results: A representative sample of the students answered that cancer can affect oral tissues and showed mastery over the role of cigarette as a risk factor, however, they were deficient in demonstrating the most affected age group. All groups presented critical knowledge levels, opposed to selfevaluation questions. There was a tendency of first and second course year to settle more questions. Conclusion: The data obtained suggest the need for strategies directed to the teaching/learning process, aiming the best use and establishment of knowledge about oral cancer, as well as the focus on the development of prevention policies for oral cancer in the pedagogical project of the evaluated courses.
\end{abstract}

Descriptors: Mouth Neoplasms; Oral Health; Area Health Education Centers; Educational Measurement.

\section{Resumen}

Objetivo: Evaluar el nivel de conocimiento de estudiantes de diferentes cursos del área de salud de la Universidad Vale do Rio Verde de Três Corações (Minas Gerais, Brasil) sobre cáncer oral. Método: Fue realizado un estudio observacional, en el cual 314 estudiantes de los cursos de farmacia, enfermería, nutrición y odontología respondieron un cuestionario estructurado con 17 preguntas divididas entre auto-evaluación y características objetivas sobre cáncer oral. Resultados: Una parte representativa de la población universitaria sabe que el cáncer puede afectar la cavidad oral y que el tabaquismo y alcoholismo son factores de riesgo, sin embargo, mostraron desconocimiento sobre el grupo etario más afectado. Se observó que los 4 cursos presentaron un nivel de conocimiento deficiente, contraponiéndose a los datos obtenidos del cuestionario de auto-evaluación. Hubo una tendencia de los alumnos de primer y segundo año de pregrado a obtener puntuación mayor. Conclusión: Los datos obtenidos sugieren la necesidad de estrategias direccionadas al proceso enseñanza/aprendizaje, apuntando al mejor aprovechamiento y fijación del conocimiento acerca del cáncer oral, así como al enfoque en el desarrollo de políticas de prevención de esta enfermedad en el proyecto pedagógico de estos cursos.

Descriptores: Neoplasias de la Boca; Salud Bucal; Centros Educacionales de Áreas de Salud; Evaluación Educacional.

\section{INTRODUÇÃO}

O câncer de boca é uma doença multifatorial que pode afetar lábios, orofaringe e cavidade oral. É o sexto tipo de câncer mais incidente no mundo. No Brasil, estima-se a ocorrência de 11.140 novos casos em homens e 4.350 em mulheres, em $2016^{1}$. Sendo que, em geral, o tratamento do câncer de boca é oneroso, devido aos complexos métodos de 
reabilitação. Uma vez que esta doença é diagnosticada, muitas vezes, em fases tardias, compõe uma das principais causas de óbito por neoplasias no mundo ${ }^{1-3}$.

O carcinoma de células escamosas (CCE) é o tipo mais comum de neoplasia maligna a afetar os tecidos orais, compondo de 90 a $95 \%$ dos casos. Inicialmente, pode apresentar-se assintomático ou, ainda, mimetizar condições benignas comuns da boca ${ }^{2}$. O diagnóstico precoce, assim como em outras neoplasias, é muito importante para maior eficiência no tratamento e melhoria na qualidade de vida e sobrevida do paciente. Algumas lesões podem preceder o carcinoma de células escamosas, como, por exemplo, a leucoplasia e a eritroplasia. Idealmente, o diagnóstico dessas lesões não deveria ser negligenciado, uma vez que, bem diagnosticadas e tratadas, impossibilitam a progressão da alteração celular e tecidual, por consequência, inviabilizam a ocorrência de lesões malignas passíveis de prevenção ${ }^{4}$. O deficiente reconhecimento de lesões potencialmente malignizáveis e lesões malignas em fases iniciais, por parte da população e por profissionais da área da saúde, tem sido apontada como um dos principais fatores que podem contribuir para o diagnóstico tardio do câncer de boca ${ }^{5-8}$.

A detecção tardia da doença reflete a deficiência de treinamento, bem como de educação continuada, que visem o aperfeiçoamento dos profissionais acerca dos sinais e sintomas iniciais no desenvolvimento da doença ${ }^{9-10}$. O CCE representa uma causa importante de morbimortalidade e é considerada como um dos maiores problemas de saúde pública, uma vez que mais de $50 \%$ dos casos são diagnosticados em estágio avançado ${ }^{6-11}$. A deficiência no diagnóstico precoce resultará na invasão de estruturas vizinhas, na qual é possível observar que cerca de $40 \%$ dos pacientes portadores de câncer de boca morrem pela incapacidade de controle locorregional da doença e na formação de metástases linfonodais e à distância, acarretando em uma taxa de óbito em razão de CCE de aproximadamente $24 \%{ }^{2}$.

O tabagismo é o principal fator de risco para o desenvolvimento destas neoplasias na cavidade oral, sendo potencializado pelo consumo de bebidas alcoólicas, essa associação de hábitos pode elevar para 35 vezes as chances de desenvolvimento da doença ${ }^{9}$. A prevenção primária desta condição está relacionada aos fatores etiológicos e nas alterações do estilo de vida ${ }^{2,12}$. Alguns fatores de risco predispõe a manifestação do CCE em partes distintas da cavidade oral, como a exposição à radiação UVA/UVB no lábio inferior ${ }^{13}$ e a contaminação pelo Human Papiloma Vírus - HPV manifestando lesões em orofaringe ${ }^{14}$.

É de fundamental importância que a população tenha acesso à informação sobre a prevenção dos cânceres. Cerca de um terço das neoplasias malignas mais comuns podem ser evitadas através de hábitos saudáveis, como não fumar, redução no consumo de álcool (principalmente concomitante ao tabagismo), prática de atividades físicas regulares e alimentação balanceada ${ }^{1}$.

Os acadêmicos da área de saúde compõem a população que constituem a base do conhecimento e devem ter sua formação direcionada, de modo a evitar as dificuldades no reconhecimento precoce da doença ${ }^{12,15}$. Alguns trabalhos avaliam o conhecimento de acadêmicos de odontologia sobre o $\mathrm{CCE}^{4,8}$. Tendo em vista a existência de poucos estudos envolvendo os acadêmicos de outras áreas da saúde $\mathrm{e}^{10,16,17}$, o presente estudo teve por objetivo avaliar o conhecimento sobre o câncer de boca entre os acadêmicos dos cursos de graduação $m$ áreas da saúde da UNINCOR de Três Corações - MG.

\section{MATERIAL E MÉTODO}

O presente estudo foi submetido e aprovado pelo Comitê de Ética em Pesquisa da Universidade Vale do Rio Verde - Três Corações/ MG (CAAE:57769516.8.0000.5158). A amostra foi composta por acadêmicos regularmente matriculados em todos os períodos dos cursos de graduação em: Farmácia, Enfermagem, Nutrição e Odontologia.

Os participantes foram abordados pelo examinador a participar através de um questionário estruturado com 18 perguntas, divididos em questões de auto avaliação e questões objetivas acerca do câncer de boca, com embasamento em estudos prévios que avaliaram o conhecimento de alunos e profissionais da saúde sobre o tipo de câncer oral mais comum, local mais acometido, fatores de risco, manifestações clínicas das lesões, gênero e faixa etária mais acometidos $5,8,18,19$.

As informações coletadas a partir dos questionários foram transcritas para um banco de dados (Microsoft Excel ${ }^{\circledR}$ - Microsoft Corporation) e analisados estatisticamente com o suporte do software SPSS ${ }^{\circledR} 20$ for Windows (Statistical Package for Social Sciences). Os dados foram analisados e os resultados foram expressos em porcentagem para a auto avaliação e para as questões objetivas sobre o câncer de boca. As respostas do questionário objetivo foram comparadas através do teste $t$, afim de comparar as médias de acertos entre anos e entre cursos abordados. A percepção da importância do conhecimento do câncer oral explicitadas por uma escala visual foi analisada através da média numérica obtida nos questionários.

\section{RESULTADOS}

Participaram do estudo 314 acadêmicos de cursos da área da saúde da UNINCOR - MG, sendo 165 alunos do curso de odontologia (52,5\%), 37 do curso de farmácia $(11,8 \%), 68$ do curso de nutrição $(21,7 \%)$ e 44 do curso de enfermagem (14\%). A maioria dos alunos eram do gênero feminino $(71,7 \%)$. A média de idade foi de 23,04 anos e a maior parte dos estudantes encontra-se no $1^{\circ}$ ano dos cursos da saúde (128 alunos). A distribuição e perfil dos acadêmicos participantes é ilustrada de acordo com a Tabela 1.

Tabela 1. Distribuição e perfil dos participantes

\begin{tabular}{|c|c|c|c|c|c|c|c|}
\hline & \multirow{2}{*}{$\begin{array}{c}\text { Idade } \\
\text { Média } \pm \\
\text { DP }\end{array}$} & \multirow{2}{*}{$\begin{array}{c}\text { Gênero } \\
\text { F/M }\end{array}$} & \multicolumn{5}{|c|}{$\begin{array}{l}\text { Participantes por período da } \\
\text { graduação (n) }\end{array}$} \\
\hline & & & $\begin{array}{c}10 \\
\text { ano }\end{array}$ & $\begin{array}{c}2^{\circ} \\
\text { ano }\end{array}$ & $\begin{array}{c}3^{\circ} \\
\text { ano }\end{array}$ & $\begin{array}{c}4^{\circ} \\
\text { ano }\end{array}$ & $\begin{array}{l}5^{\circ} \\
\text { ano }\end{array}$ \\
\hline \multirow{2}{*}{ Odontologia } & $165(52,5 \%)$ & $105 \mathrm{~F}(63,6 \%)$ & \multirow{2}{*}{46} & \multirow{2}{*}{43} & \multirow{2}{*}{45} & \multirow{2}{*}{31} & \multirow{2}{*}{0} \\
\hline & $23 \pm 0$ & $60 \mathrm{M}(36,4 \%)$ & & & & & \\
\hline \multirow{2}{*}{ Farmácia } & $37(11,8 \%)$ & $31 \mathrm{~F}(83,8 \%)$ & \multirow{2}{*}{20} & \multirow{2}{*}{17} & \multirow{2}{*}{0} & \multirow{2}{*}{0} & \multirow{2}{*}{0} \\
\hline & $21 \pm 1$ & $6 \mathrm{M}(16,2 \%)$ & & & & & \\
\hline \multirow{2}{*}{ Nutrição } & $68(21,7 \%)$ & $33 \mathrm{~F}(75 \%)$ & \multirow{2}{*}{28} & \multirow{2}{*}{16} & \multirow{2}{*}{0} & \multirow{2}{*}{0} & \multirow{2}{*}{0} \\
\hline & $24 \pm 1$ & $11 \mathrm{M}(25 \%)$ & & & & & \\
\hline \multirow{2}{*}{ Enfermagem } & $44(14 \%)$ & $56 \mathrm{~F}(82,4 \%)$ & \multirow{2}{*}{34} & \multirow{2}{*}{0} & \multirow{2}{*}{20} & \multirow{2}{*}{1} & \multirow{2}{*}{13} \\
\hline & $23 \pm 1$ & $12 \mathrm{M}(17,6 \%)$ & & & & & \\
\hline \multirow[t]{2}{*}{ TOTAL } & 314 & $225 \mathrm{~F}(71,7 \%)$ & \multirow[t]{2}{*}{128} & \multirow[t]{2}{*}{76} & \multirow[t]{2}{*}{65} & \multirow[t]{2}{*}{32} & \multirow[t]{2}{*}{13} \\
\hline & $23,04 \pm 5,19$ & $89 \mathrm{M}(28,3 \%)$ & & & & & \\
\hline
\end{tabular}

Na primeira parte do questionário, voltada a auto avaliação sobre o conhecimento adquirido acerca do câncer de boca, 298 acadêmicos $(94,9 \%)$ relataram ter embasamento sobre o tema, no entanto, 36,6\% dos entrevistados avaliaram seu nível de conhecimento como razoável e $26,4 \%$ relatam que seu conhecimento sobre o assunto era insuficiente. A maior parte dos entrevistados $(60,2 \%)$ afirmou ter adquirido tal conhecimento durante a 


\section{DISCUSSÃO}

O carcinoma de células escamosas é uma neoplasia maligna que surge a partir da proliferação descontrolada que ocorre a partir de uma célula geneticamente alterada proveniente do epitélio pavimentoso estratificado paraqueratinizado, que reveste a cavidade oral, orofaringe e lábios. O epitélio de revestimento é exposto a vários agentes com potencial carcinogênico, como, por exemplo, substâncias tóxicas presentes no cigarro (tecidos intrabucais) ou raios ultravioletas (principalmente no lábio inferior). As agressões físicas ou químicas alteram a célula geneticamente, propiciando sua proliferação clonal descontrolada. As primeiras alterações no tecido epitelial podem apresentar-se clinicamente como regiões brancas, vermelhas ou mistas, chamadas de leucoplasia, eritroplasia ou eritroleucoplasia, respectivamente nos tecidos intraorais, já em lábio, é possível notar a presença de displasia epitelial em graus variados, associada a elastose solar, imediatamente abaixo do epitélio, chamada de queilite actínica. Essas alterações são restritas ao epitélio e não compõem, de início, uma neoplasia verdadeira, por este motivo, são classificadas como lesões potencialmente malignizáveis ${ }^{20}$. Quando a alteração epitelial atinge todas as camadas epiteliais (camada basal, espinhosa e granulosa), pode-se classificar a lesão como um carcinoma in situ. Seguindo a progressão natural da doença, há a infiltração de tecido epitelial para dentro do córion adjacente, havendo rompimento da membrana basal, na qual, já é possível classificar como carcinoma invasivo. Quanto mais profundo forem notadas ilhas tumorais, com invasão perineural, perivascular e intramuscular, pior tende a ser o prognóstico. Devido a isso, é de extrema importância que o diagnóstico seja realizado em etapas iniciais da progressão da doença, visando um tratamento eficiente e aumento na qualidade de vida dos pacientes ${ }^{21}$.

Alguns estudos reportam o conhecimento de acadêmicos sobre o câncer de boca ${ }^{2,6,19}$. A média de idade dos participantes deste estudo foi semelhante a encontrada em outros trabalhos do mesmo gênero ${ }^{6,8,9}$. Os alunos do curso de odontologia compunham a maior parte dos participantes, pelo fato de haver uma maior quantidade de alunos e interesse por parte deste ramo do conhecimento sobre o diagnóstico precoce e demais questões pertinentes ao tema, corroborando com os achados de Awan et al. ${ }^{10}$ e Shrestha et al. ${ }^{6}$.

No presente estudo observou-se um maior índice de acertos nos segundos e terceiros anos, assim como os resultado de Dumitrescu et al. ${ }^{8}$ e Oliveira et al. ${ }^{12}$. Considerando que a maioria dos entrevistados relatou que obteve este conhecimento no curso de graduação atual, sugere-se que este resultado se deve ao fato de haver a disciplina de patologia geral e específica no plano de ensino destes cursos, na qual alguns fatores de risco para o desenvolvimento do câncer, inclusive o CCE, são abordados com maior profundidade, além de muitos alunos não reconhecerem que o atendimento multidisciplinar é um fator importante para o diagnóstico precoce.

A maioria dos alunos afirmou ter ciência sobre o câncer de boca. No entanto, no questionário de auto avaliação este conhecimento foi julgado como razoável e insuficiente por grande parte dos acadêmicos, assim como os resultados encontrado por Akbari et al. ${ }^{4}$ e Shrestha et al. ${ }^{6}$ Nos estudos de Awan et al. ${ }^{10}$ e Pinheiro et al. ${ }^{9}$ os resultados determinaram índices superiores de acerto avaliando o conhecimento desta patologia do que os índices gerais resultantes do presente trabalho. A maioria dos acadêmicos do presente estudo relatou que adquiriram conhecimento sobre o assunto durante o curso de graduação em andamento, corroborando com $\mathrm{Dib}^{5}$ e Dumitrescu et al. ${ }^{8}$. Adicionalmente, Pinheiro et al. ${ }^{9}$ identificaram deficiências na captação e fixação do aprendizado durante o curso de graduação entre os acadêmicos entrevistados.

As células normais da mucosa oral são diploides e o material genético é continuamente reparado. O tabaco possui muitas substâncias nocivas aos tecidos biológicos, como nicotina, agentes fenólicos e metais pesados ${ }^{22}$. Fumantes ativos têm contato direto e contínuo com essas substâncias, que, em associação com o tempo de uso, podem criar uma ampla área geneticamente modificada. Uma vez que o sistema de reparo não consegue bloquear a proliferação de células geneticamente modificadas, tem-se uma probabilidade aumentada de desenvolvimento de tumores. Em estudo realizado utilizando técnicas histoquímicas que envolvem citologia esfoliativa, foi demonstrado que células epiteliais de pacientes fumantes apresentam maior quantidade de aneuploidia, além de que as células apresentaram aumento na relação núcleo-citoplasma, aumento na quantidade de células queratinizadas e aumento na taxa de proliferação celular ${ }^{23}$.

Alguns autores demonstraram bom nível de conhecimento dos acadêmicos em relação aos fatores de risco. O tabagismo e etilismo foram identificados como os principais facilitadores para câncer de boca em seus estudos $^{6,9,18}$. No presente trabalho, houve deficiência no conhecimento sobre este fator, semelhante aos resultados encontrados por Lima et al. ${ }^{2}$ e Awan et al. ${ }^{10}$, comprometendo diretamente as informações prestadas ao grande público. $\mathrm{O}$ tabagismo é uma prática antiga, mas que, apesar das campanhas públicas alertando sobre seus riscos, apresenta, ainda, muitos adeptos. Pacientes tabagistas devem ser acompanhados de perto quanto à possíveis alterações na mucosa, além da orientação sobre os malefícios da prática ${ }^{22}$.

O HPV (Human Papilloma Virus) é um vírus pequeno, com DNA de dupla hélice, não envelopado, epiteliotrópico que pertence à família papillomaviridae. O vírus recebe este nome, pois alguns tipos de HPV causam verrugas e/ou papilomas, que são lesões proliferativas nãoneoplásicas. Alguns subtipos de HPV apresentam alto risco para o desenvolvimento de neoplasias, como os tipos 16, 18, 31 e 33. Estes subtipos são conhecidos por infectar células epiteliais e expressarem proteínas que alteram as vias de supressão tumoral pela inativação do gene p53 e pRb (retinoblastoma). Recentemente, os subtipos 6 e 11 do HPV foram identificados em CEE orais e os subtipos 6, 16 e 18 foram identificados em lesões potencialmente malignizáveis orais $^{24}$. Adicionalmente, de todos os CEE orais com coinfecção por HPV, o subtipo 16 foi identificado em $90 \%$ da amostra, enquanto que, no total, 50\% dos CEE orais apresentaram infecção com algum tipo de HPV. Logo, a relação entre HPV e câncer de boca já está estabelecida na literatura ${ }^{14}$. Quando questionados sobre esta possível interconexão, aproximadamente metade dos acadêmicos $(51,6 \%)$ afirmaram que o HPV pode estar relacionado na patogenia do câncer de boca. Em relação à radiação UV, as respostas apresentaram índice maior de acerto, sendo que estes resultados corroboram com os resultados de Dumitrescu, Ibric ${ }^{8}$, porém confrontam os resultados de Alhazzazi ${ }^{19}$, que, mesmo em períodos semelhantes de avaliação, obtiveram resultados inferiores aos encontrados neste trabalho.

De acordo com os resultados de $\mathrm{Dib}^{5}$ e Razavi et al. ${ }^{11}$, a maioria da população entrevistada encontra-se empenhada com relação à importância do conhecimento 
desta doença, incluindo o diagnóstico precoce, encaminhamento profissional e tratamento. No entanto, apresentaram níveis de acerto muito baixos, que demonstram deficiência no processo ensino-aprendizagem nos cursos em questão.

O presente estudo apresenta como limitação, o fato de ter sido realizado em uma única instituição de ensino. Nessa instituição, apenas o curso de enfermagem possui duração mínima de 5 anos. Os demais cursos possuem duração mínima de 4 anos, sendo que o curso de nutrição, por ser novo na grade, ainda não concluiu a formação da primeira turma do curso.

Existe uma necessidade urgente de otimizar a capacidade dos profissionais de saúde a realizar a detecção precoce do CCE. O estudo permitiu uma avaliação criteriosa do nível de conhecimento sobre câncer bucal, possibilitando a adoção de medidas educacionais continuadas ao longo dos cursos de graduação, a fim de melhorar a retenção de conhecimento destes alunos com relação ao comportamento desta patologia. Informações como as apresentadas neste estudo são de extrema importância, pois fornecem subsídios para que haja aprimoramento do projeto pedagógico dos cursos relacionados, a fim de melhorar o processo ensinoaprendizagem, consequentemente, melhorar o perfil do novo profissional, por consequência, melhoria na qualidade da informação passada ao grande público.

\section{CONCLUSÃO}

De acordo com os dados encontrados neste estudo, é possível inferir a necessidade do desenvolvimento de estratégias que promovam melhorias referentes ao processo de ensino e aprendizagem que maximizem o conhecimento dos futuros profissionais da saúde acerca do câncer de boca, para que, além de estarem aptos a diagnosticarem precocemente possíveis lesões malignas, possam ter embasamento suficiente para instruir, de maneira adequada, a população ou fazer o correto encaminhamento do paciente, melhorando o prognóstico dos pacientes portadores de CCE.

\section{REFERÊNCIAS}

1. INCA. Estimativa 2016 - Incidência de Câncer no Brasil. Rio de Janeiro: Coordenação de Prevenção e Vigilância; 2016. p. 122

2. Lima AAS de, França BHS, Ignácio SA, Baioni CS. Conhecimento de alunos universitários sobre câncer bucal. Rev Bras Cancerol. 2005; 51(4):283-8.

3. Martins JD, Oliveira J, Andrade M. Determinantes sociais de saúde e a ocorrência de câncer oral: uma revisão sistemática de literatura. Rev salud pública. 2014; 16(5):786-98.

4. Akbari N, Raeesi V, Khazaei T, Ramezanzadeh K, Ebrahimipour S. Evaluation of general dentists' and dental specialists' knowledge about oral cancer in South Khorasan-Iran 2014. Asian Pac J Cancer Prev. 2015; 16(16):6987-90.

5. Dib LL. Evaluation of the knowledge about oral cancer among undergraduate dental students of different units at University Paulista. Rev Inst Ciênc Saúde. 2005; 23(4):287-96.

6. Shrestha A, Marla V, Shrestha S, Agrawal D. Awareness of undergraduate dental and medical students towards oral cancer. J Canc Educ. 2016. https://doi.org/10.1007/s13187-016-1023-4
7. Falcão MML, Alves TDB, Freitas VS, Coelho TCB. Conhecimento dos cirurgiões-dentistas em relação ao câncer bucal. RGO. 2010; 58(1):27-33.

8. Dumitrescu AL, Ibric S, Ibric-Cioranu V. Assessing Oral Cancer Knowledge in Romanian Undergraduate Dental Students. J Cancer Educ. 2014; 29(3):506-13.

9. Pinheiro SMS, Cardoso JP, Prado FO. Conhecimentos e Diagnóstico em Câncer Bucal entre Profissionais de Odontologia de Jequié, Bahia. Rev Bras Cancerol. 2010; 56(2):195-205.

10. Awan KH, Khang TW, Yee TK, Zain RB. Assessing oral cancer knowledge and awareness among Malaysian dental and medical students. J Cancer Res Ther. 2014; 10(4):903-7.

11. Razavi SM, Zolfaghari B, Foroohandeh M, Doost ME, Tahani B. Dentists' knowledge, attitude, and practice regarding oral cancer in Iran. J Cancer Educ. 2013; 28(2):335-41.

12. Oliveira JMB de, Pinto LO, Lima NGM, Almeida GCM de. Câncer de Boca: Avaliação do Conhecimento de Acadêmicos de Odontologia e Enfermagem quanto aos Fatores de Risco e Procedimentos de Diagnóstico. Rev Bras Cancerol. 2013; 59(2):2011-8

13. Warnakulasuriya S. Global epidemiology of oral and oropharyngeal cancer. Oral Oncol. 2009; 45(45):309-16.

14. Liang X-H, Lewis J, Foote R, Smith D, Kademani D. Prevalence and significance of human papillomavirus in oral tongue cancer: the Mayo Clinic experience. J Oral Maxillofac Surg. 2008; 66(9):1875-80.

15. Pentenero M, Chiecchio A, Gandolfo S. Impact of academic and continuing education on oral cancer knowledge, attitude and practice among dentists in north-western Italy. J Cancer Educ. 2014; 29(1):151-7.

16. Kujan O, Abuderman A, Azzegahiby S, Alenzi FQ, Idrees M. Assessing oral cancer knowledge among Saudi medical undergraduates. J Cancer Educ. 2013; 28(4):717-21.

17. Carter LM, Ogden GR. Oral cancer awareness of undergraduate medical and dental students. BMC Med Educ. 2007; 7:44.

18. Gomes SV, Conceição TS, Neves PAM, Lopes MFF, Cruz CFN da. Knowledge on oral cancer among dentistry students at Federal University of Maranhão. Rev Odontol UNESP. 2015; 44(1):44-50.

19. Alhazzazi TY. Evaluation of Head and Neck Cancer Awareness and Screening Status in Jeddah, Saudi Arabia. Asian Pac J Cancer Prev. 2016; 17(3):1135-9.

20. van der Waal I, Schepman KP, van der Meij EH, Smeele LE. Oral leukoplakia: A clinicopathological review. Oral Oncol. 1997; 33(5):291-301.

21. Neville BW, Damm D, Allen C, Bouqot J. Patologia Oral e Maxilofacial. 3 ed. Elsevier: Rio de Janeiro; 2009. $992 \mathrm{p}$.

22. Andreotti G, Freedman ND, Silverman DT, Lerro CC, Koutros S, Hartge P, et al. Tobacco use and cancer risk in the Agricultural Health Study. Cancer Epidemiol Biomarkers Prev December 292016 DOI:10.1158/10559965.EPI-16-0748.

23. Souto GR, Caliari MV, Lins CEC, De Aguiar MCF, De Abreu MHNG, Mesquita RA. Tobacco use increase the number of aneuploid nuclei in the clinically healthy oral epithelium. J Oral Pathol Med. 2010; 39(8):605-10.

24. Khot KP, Deshmane S, Choudhari S. Human papilloma virus in oral squamous cell carcinoma - the enigma unravelled. Chin J Dent Res. 2016; 19(1):17-23. 


\section{CONFLITO DE INTERESSES}

Os autores declaram não haver conflitos de interesse.

\section{AUTOR PARA CORRESPONDÊNCIA}

Matheus Henrique Lopes Dominguette matheus.dominguete@unincor.edu.br

Submetido em 26/04/2017

Aceito em 06/06/2017 